\title{
Synthesis, characterization and in vitro biological screening of 4-hydroxy naphthalen-1-yl, naphtho[1,2-b]furan, benzo $[h]$ chromene and 5,6-dihydropyridazine derivatives containing sulfonamide moiety
}

\author{
Mohamed S. A. El-Gaby ${ }^{1, *}$, Mohamed I. Hassan ${ }^{1, *}$, Modather F. Hussein ${ }^{1}$, Ahmed M. Ali ${ }^{1}$, Miral A. \\ Abdelmoaz $^{2}$, Mahmoud M. Elaasser ${ }^{3}$, Faraghally A. Faraghally ${ }^{1}$ \\ ${ }^{1}$ Department of Chemistry, Faculty of Science, Al-Azhar University at Assiut, Assiut 71524, Egypt \\ ${ }^{2}$ Pharmaceutical Organic Chemistry, Faculty Pharmacy, Sinai University, Kantara, Egypt \\ ${ }^{3}$ Regional Center for Mycology \& Biotechnology, Al-Azhar University, Cairo, Egypt
}

\begin{abstract}
In this study, a series of 4-((4-hydroxynaphthalen-1-yl)diazenyl)benzenesulfonamides have been prepared by subsequent diazotization of sulfonamide derivatives and coupling with 1-naphthol in alkaline medium. Cyclization of 4-((4-hydroxynaphthalen-1-yl)diazenyl)benzenesulfonamides with cinnamic acid in the presence of a basic catalyst afforded the novel naphtho[1,2-b]furans. Also, 4-((4-hydroxynaphthalen-1-yl)diazenyl)benzenesulfonamides can be cyclized with $\alpha$-cyanocinnamonitriles to afford 2-amino-3-cyano-4-phenyl-4H-benzo[ $h]$ chromenes. 4-(4-amino-3,5dicyano-6-iminopyridazin-1(6H)-yl)benzenesulfonamides were obtained at room temperature by treatment of 2-amino-1,1,3-tricyanopropene with a diazonium salt of sulfonamide derivatives. The structures of newly synthesized compounds were confirmed by analytical data and spectroscopic techniques. The antimicrobial activity of the obtained compounds was assessed in vitro by qualitative and quantitative (minimum inhibitory concentration) (MIC) assays.
\end{abstract}

Keywords: sulfonamide; azobenzene; naphtho[1,2-b]furan; benzo[h]chromene; pyridazine.

\section{Introduction}

Substituted azobenzene have attracted considerable attention based on their various physical and chemical properties, such as bright colors, good stability, low flammability, and rapid, reversible photo-isomerization 1-4. Aromatic azo compounds are widely used in the chemical industry as dyes, pigments ${ }^{5,6}$, food additives 7 , indicators ${ }^{3}$, radical reaction initiators ${ }^{8}$ and therapeutic agents ${ }^{9,10}$. Also, azobenzenes have shown promising applications in photo-optical media ${ }^{11}$, photoswitches ${ }^{12}$, photo-mechanical systems ${ }^{13}$, micro patterning ${ }^{14}$, nonlinear optical media ${ }^{15}$, molecular shuttles ${ }^{16}$, nanotubes ${ }^{17}$, and in the manufacture of protective eye glasses and filters ${ }^{18}$.
Naphthofuran derivatives have also been reported to possess diverse biological activities, including antityrosinase, antioxidant and antibacterial 19. Naphtho[1,2-b]furans are very important structural units found in diverse natural and synthetic products 20 . They possess a broad spectrum of biological activities and have been used as precursors for the synthesis of bioactive materials. Naphtho[1,2-b]furan-4,5-dione (NFD, Fig. 1), a 1,2-furanonaphtho quinone, was originally isolated from avicennia marina belonging to the family Avicenniaceae and can be synthesized by a chemical process. NFD was found to show potent cytotoxicity against human cancer cell lines, including KB (human epidermoid carcinoma, IC50 = 3.05 \pm 0.195 $\mu \mathrm{M}$ ), HeLa (human cervical carcinoma, IC50 = $2.85 \pm$ $0.210 \mu \mathrm{M}$ ) and HepG2 (human hepatocellular carcinoma, IC50 $=3.00 \pm 0.040 \mu \mathrm{M}$ ) cell lines ${ }^{21}$.
*Corresponding authors: Mohamed S. A. El-Gaby, Mohamed I. Hassan

Email address: m_elgaby@hotmail.com,mhi211978@gmail.com

DOI: http://dx.doi.org/10.13171/mjc751912061355msaeg
Received October 11, 2018

Accepted November 10, 2018

Published December 6, 2018 
Recently, Chen et al. 22 discover, $N$-(naphtha [1,2-b]furan-5-yl) benzene sulfonamides (Fig. 1), as novel selective inhibitors of triple-negative breast cancer (TNBC).

Chromene derivatives are very an important class of heterocyclic compounds, widely distributed in natural products. Chromene and its derivatives have also been recognized as one type of 'privileged medicinal scaffolds' due to their unique pharmacological and biological activities ${ }^{23}$. Dong et al. ${ }^{24,25}$ designed and prepared a series of 4-amino- $2 H$-benzo $[h]$ chromen-2-one and 4-amino-7,8,9,10-tetrahydro- $2 \mathrm{H}$ benzo[ $h]$ chromen-2-one derivatives based on the potent anticancer agents neo-tanshinlactone and its 4-ethyl analogue $^{26}$.

Sulfonamides are another important compounds family for the medicinal industry, and they are now extensively used drugs for the treatment or conservation of different illnesses ${ }^{27}$. In clinical medicine, they have been used as anticancer 28, antimicrobial ${ }^{29}$, antiobesity ${ }^{30}$, carbonic anhydrase 31 and acetylcholinesterase inhibitor agents for Alzheimer's disease ${ }^{32}$.

In view of the above-mentioned benefits and in continuation of our interest in biologically active compounds ${ }^{33-37}$, we report herein the synthesis of some novel 4-((4-hydroxynaphthalen-1-yl)diazenyl)benzene sulfonamides3a-e, naphtho[1,2- $b]$-furans 6a-c,benzo $[h]$ chromenes8a,b and 4-(4-amino-3,5dicyano-6-imino-5,6-dihydropyridazin-1(4H)-yl)benzenesulfonamides 13a-e containing a sulfonamido moiety to evaluate their antimicrobial biological activity.<smiles>O=C1C(=O)c2ccoc2-c2ccccc21</smiles>

Naphtho[1,2-b]furan-4,5-dione (NFD)
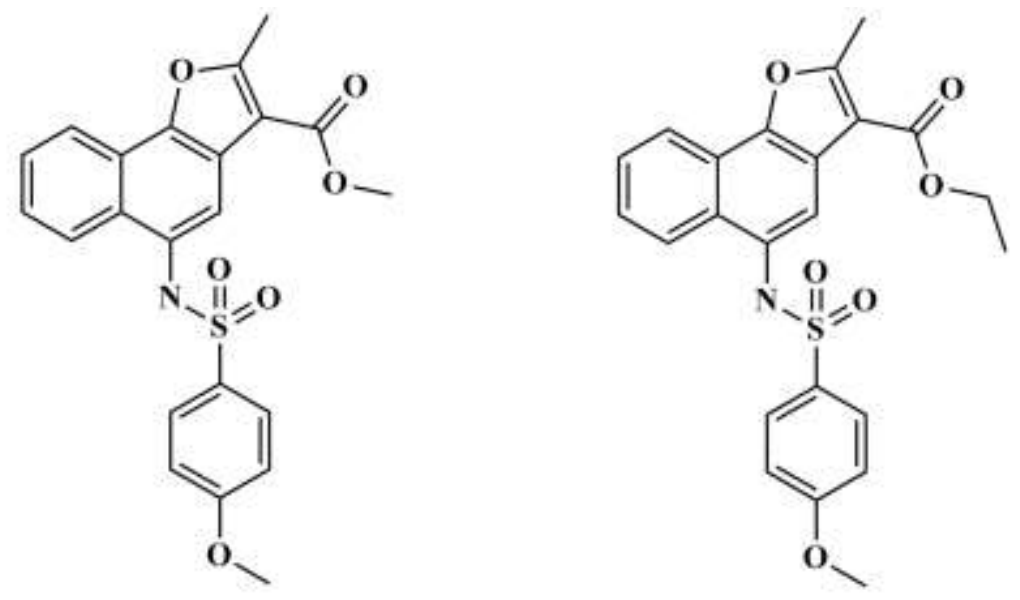

$\mathrm{N}$-(naphtho[1,2-b]furan-5-y]) benzenesulfonamides

Figure 1.

\section{Results and Discussion}

\section{Syntheses and characterizations of the compounds}

A series of 4-((4-hydroxynaphthalen-1yl)diazenyl)benzenesulfonamides 3a-e were synthesized by coupling of diazonium salt of sulfonamide derivatives 1a-e with 1-naphthol 2 in presence of $10 \%$ sodium hydroxide (Scheme 1). Diazotization was carried out in the presence of nitrosyl chloride at $0-5^{\circ} \mathrm{C}$. The structure of compounds 3a-e was determined by their elemental analysis and spectral data. Elementary analysis indicated that sulfur was present. The infrared spectra of all isolated compounds were consistent with the assumed structures. The infrared spectra of compounds 3a-e showed the presence of absorption band at 3357-3448 $\mathrm{cm}^{-1}$ which is characteristic of the hydroxyl group beside two absorptions bands for azo and sulfone groups. The representative ${ }^{1} \mathrm{HNMR}$ spectrum of compound $\mathbf{3 a}$ (DMSO-d6) shown 7.1, 7.75, 8.35, 8.80 (4d, 4H, naphtho-H), 7.47, $7.58(2 \mathrm{~m}, 2 \mathrm{H}$, naphtho-H), 7.94, 8.24 (2d, 4H, AB-system), 8.10 (s, 2H, $\mathrm{NH}_{2}$ exchangeable with $\left.\mathrm{D}_{2} \mathrm{O}\right), 12.36$ (br, $1 \mathrm{H}, \mathrm{OH}$ exchangeable with $\mathrm{D}_{2} \mathrm{O}$ ). The molecular ion peak of compound $\mathbf{3 c}$ was observed at $\mathrm{m} / \mathrm{z} 410(42.83 \%)$ corresponding to the molecular formula $\mathrm{C}_{19} \mathrm{H}_{14} \mathrm{~N}_{4} \mathrm{O}_{3} \mathrm{~S}_{2}$, and the base peak was found in the spectrum at $\mathrm{m} / \mathrm{z} 65$. The enolic- $\mathrm{OH}$ groups of all the compounds were chemically detected by the treatment with $\mathrm{FeCl}_{3}$ solution, which gives characteristic color. 


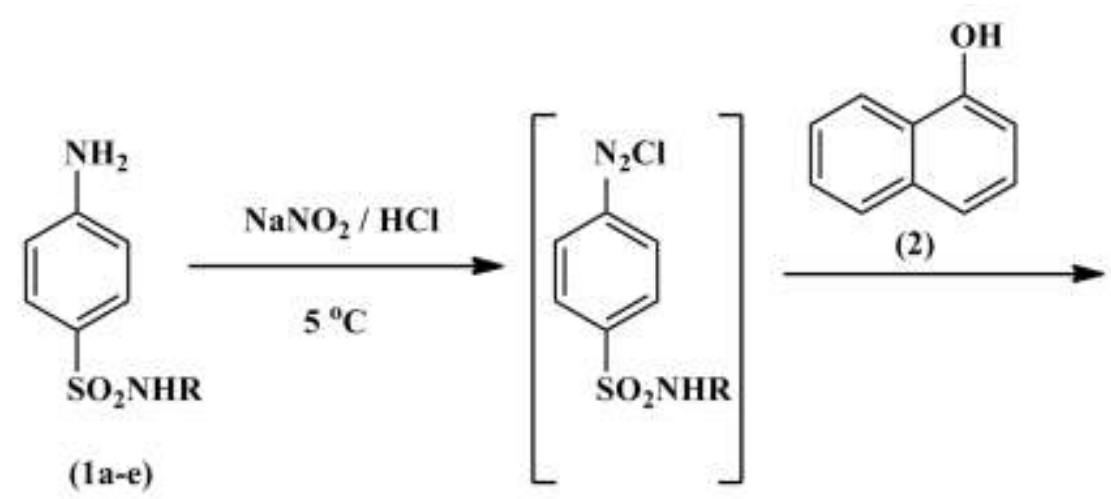

1 and 3 a; $R=H$

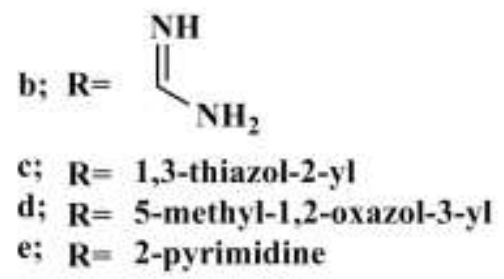<smiles>[R]NS(=O)(=O)c1ccc(N=Nc2ccc(O)c3ccccc23)cc1</smiles>

(3a-e)

Scheme 1. Synthesis of 4-((4-hydroxynaphthalen-1-yl)diazenyl)benzenesulfonamides 3a-e

The reactivity of 4-((4-hydroxynaphthalene1-yl)diazenyl)benzenesulfonamides $\mathbf{3}$ towards some carbon electrophiles was investigated. Thus, it has been found that the reaction of 4-((4-hydroxynaphthalen1-yl)diazenyl)benzenesulfonamides 3a-c with cinnamic acid 4 in refluxing $N, N$-dimethylformamide (DMF) containing catalytic amounts of piperidine gave naphtho[1,2-b]furans 6a-c rather than the expected naphthopyran 5 (Scheme 2).

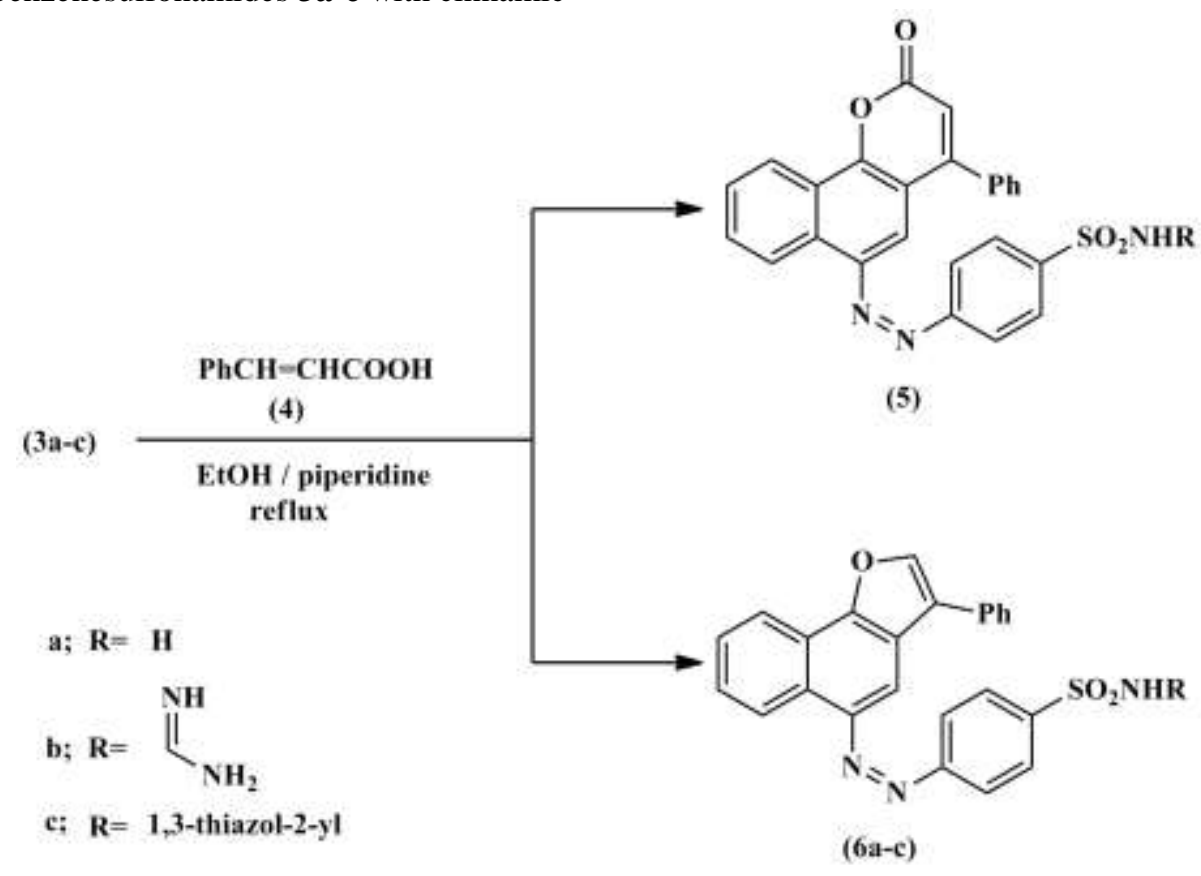

Scheme 2. Synthesis of naphtho[1,2-b]furans 6a-c 
The structures of compounds $\mathbf{6 a - c}$ were established by spectroscopic tools as well as elemental analyses data. The infrared spectra of compounds 6a-c indicated the absence of the hydroxyl and carbonyl absorption bands. The ${ }^{1} \mathrm{H}$ NMR spectrum of compound $\mathbf{6 a}$ (DMSO-d $)$ 7.25-7.62 (m, 7H, Ph-H and naphtho-H), 8.20 (s, $1 \mathrm{H}$, furan-H), 8.32, 8.39(2d, $2 \mathrm{H}$, naphtho-H), $8.45,8.50\left(2 \mathrm{~s}, 3 \mathrm{H}\right.$, naphtho- $\mathrm{H}$ and $\mathrm{NH}_{2}$, exchangeable with $\left.\mathrm{D}_{2} \mathrm{O}\right), 7.98,8.55(2 \mathrm{~d}, 4 \mathrm{H}, \mathrm{AB}$-system). The mass spectrum of compound $\mathbf{6 b}$ showed a molecular ion peak at $\mathrm{m} / \mathrm{z} 469(12.15 \%)$ compatible with molecular formula $\mathrm{C}_{25} \mathrm{H}_{19} \mathrm{~N}_{5} \mathrm{O}_{3} \mathrm{~S}$. The base peak was found in the spectrum at $\mathrm{m} / \mathrm{z} 55$. Also, the mass spectrum of compound $\mathbf{6 c}$ showed a molecular ion peak atm/z 510 (7.64\%) corresponding to the molecular formula $\mathrm{C}_{27} \mathrm{H}_{18} \mathrm{~N}_{4} \mathrm{O}_{3} \mathrm{~S}_{2}$. The molecular ion of compound $\mathbf{6 c}$ underwent fragmentation to produce a peak of $\mathrm{m} / \mathrm{z} 57$, corresponding to the base peak (Scheme 3).

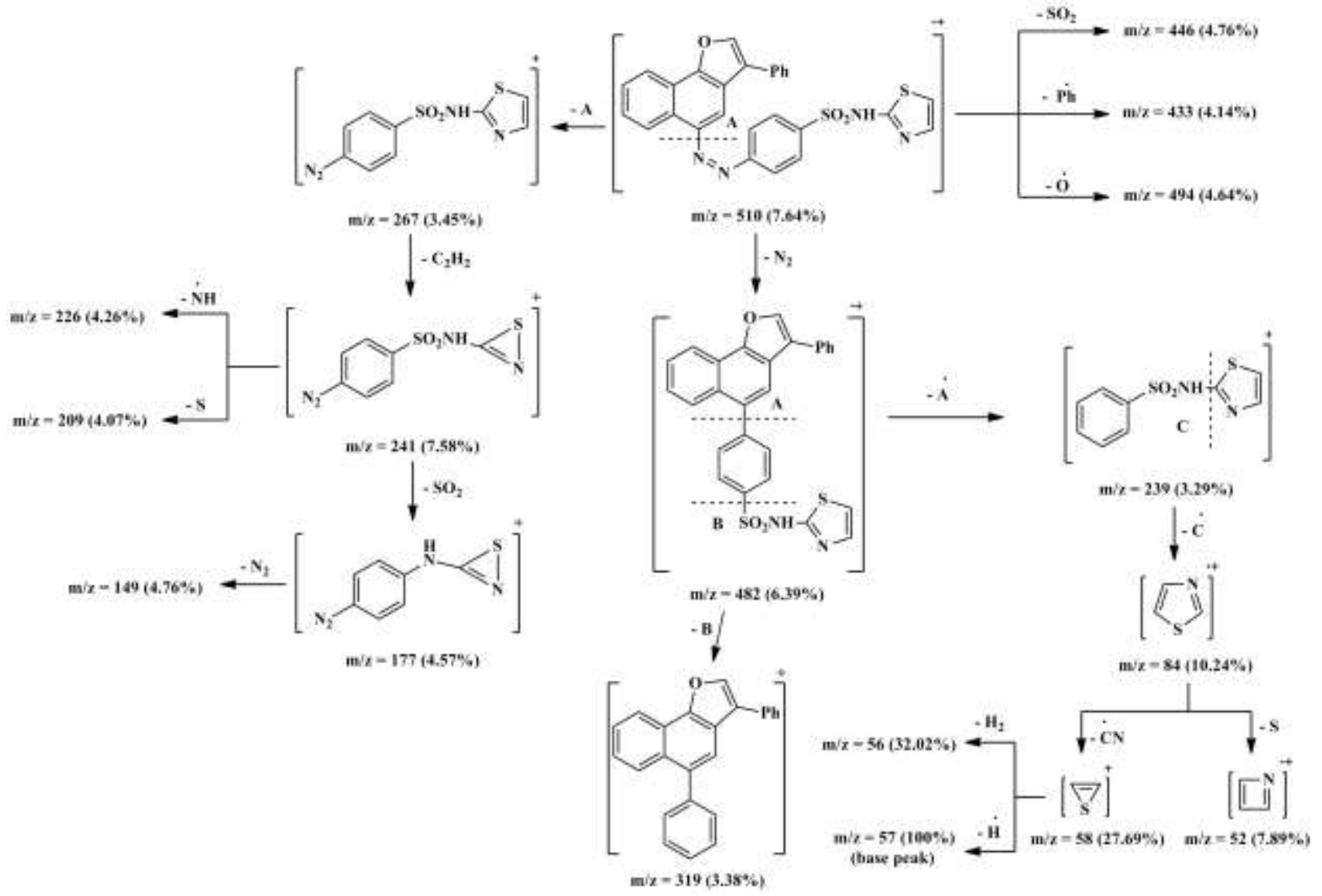

Scheme 3. Fragmentation pattern of naphtho[1,2-b]furan $\mathbf{6 c}$

The formation of 6 from the reaction of 3 with 4 is assumed to proceed via initial addition naphtholate anion (C-2) in $\mathbf{3}$ to the activated double bond in 4 to yield the non-isolable intermediate Michael adduct (A) followed by intramolecular cyclization and subsequent decarboxylation to afford the naphthofurans 6 (Scheme 4).

The reaction of compound 3a with $\alpha$ cyanocinnamonitriles was investigated. Thus, the reaction of compound 3a with $\alpha$-cyanocinnamonitriles 7 in refluxing DMF in the presence of piperidine afforded 2-amino-3-cyano-4-phenyl-4Hbenzo $[h]$ chromenes $\mathbf{8 a}, \mathbf{b}$. The structure of $\mathbf{8}$ was supported by elemental analysis and spectral data. The infrared spectra of compounds 8a, $\mathbf{b}$ displayed absorption bands for $\mathrm{NH}_{2}, \mathrm{C} \equiv \mathrm{N}, \mathrm{N}=\mathrm{N}$ and $\mathrm{SO}_{2}$ functions. The mass spectrum of compound 8a showed a molecular ion peak at $\mathrm{m} / \mathrm{z} 481$ (1.64\%) corresponding to the molecular formula $\mathrm{C}_{26} \mathrm{H}_{19} \mathrm{~N}_{5} \mathrm{O}_{3} \mathrm{~S}$. The formation of $\mathbf{8}$ from the reaction of $\mathbf{3 a}$ with 7 is assumed to proceed via initial addition of naphtholate anion (C-2) in $\mathbf{3 a}$ to the activated double bond in 7 to yield the non-isolable intermediate Michael adduct (B) followed by intramolecular cyclization through nucleophilic addition of the hydroxyl group to the cyano group and tautomerization 38 to afford benzochromene 8 (Scheme 5) . 


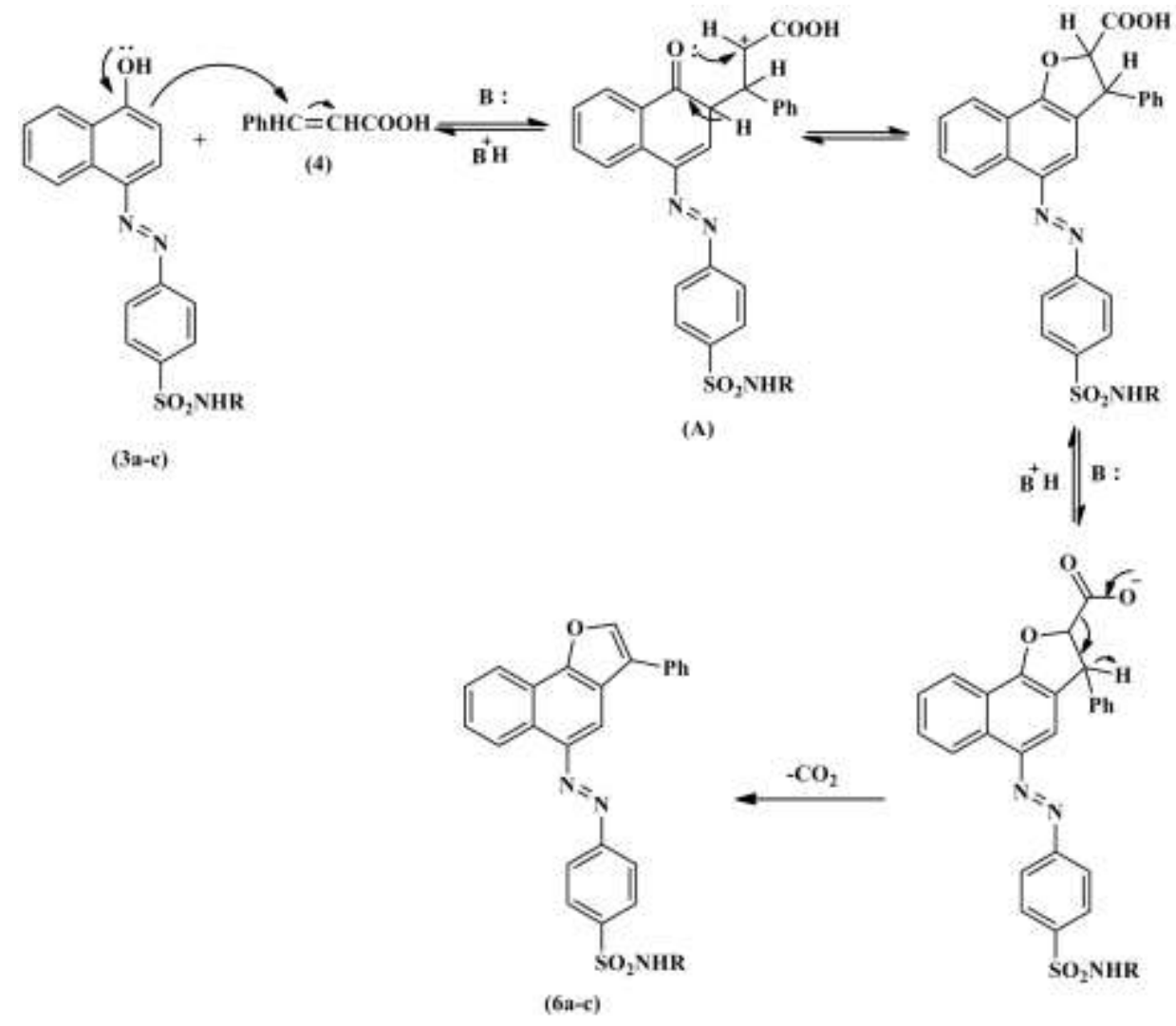

Scheme 4. Formation of naphtho[1,2-b]furans 6

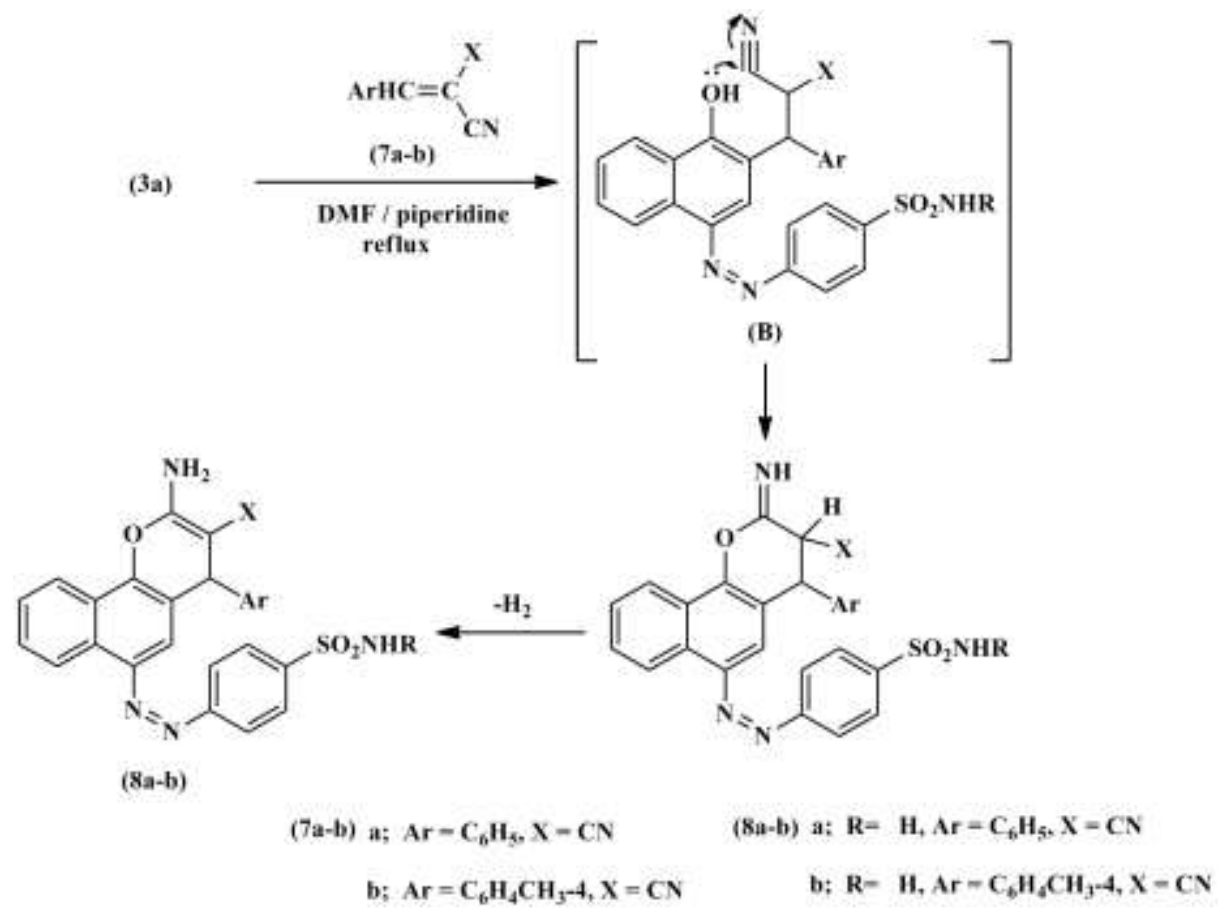

Scheme 5. 2-amino-3-cyano-4-phenyl-4H-benzo[ $h]$ chromenes 8a-b 
Pyridazine and its derivatives have been extensively investigated because of their important role especially in medicinal chemistry, a large variety of biological activities being described: antibacterial, antifungus, antituberculosis, antiviral, anti-inflammatory, anticancer, cardiovascular disorders ${ }^{39}$. Thus, treatment of 2-amino-1,1,3-tricyanopropene 9 with a diazonium

(1)

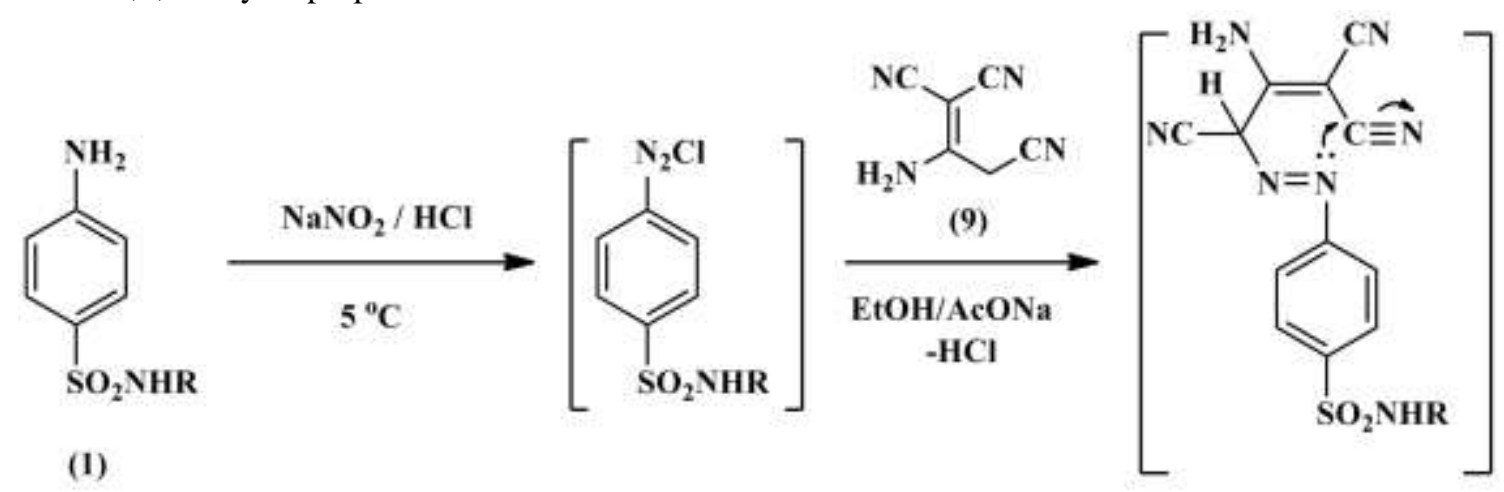

salt of sulfonamide derivatives 1a-e at room temperature gave 4-(4-amino-3,5-dicyano-6iminopyridazin-1(6H)-yl)benzenesulfonamide derivatives 11a-e, via intramolecular cyclization of $\mathbf{1 0}$ through nucleophilic addition of the nitrogen atom to the cyano group and tautomerization ${ }^{40}$ (Scheme 6).

(10)

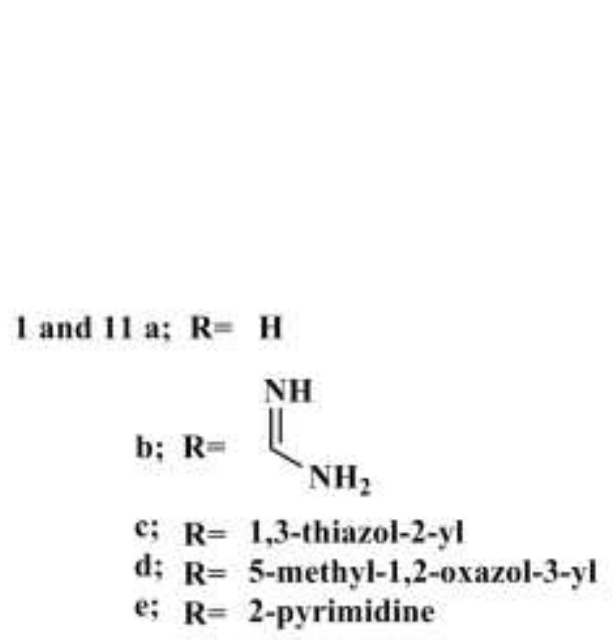

(11a-e)

Scheme 6. 4-(4-amino-3,5-dicyano-6-imino-5,6-dihydropyridazin-1(4H)- yl)benzenesulfonamides 11a-e

\section{Antimicrobial activity and minimal inhibition concentration}

The newly synthesized compounds were evaluated for their in-vitro antibacterial activity against Staphylococcus aureus, Bacillus subtilis as examples of Gram-positive bacteria, Proteus vulgarisand Escherichia coli as examples of Gram-negative bacteria, using two standard antibiotics, Ampicillin, and Gentamycin as reference drugs and antifungal potential against a representative panel of fungal strains i.e. Aspergillus fumigatus (filamentous fungi), and Candida albicans (yeast), using one standard antibiotic, Amphotericin B as reference drug. The compounds were tested for their activity at a concentration of 10 $\mathrm{mg} / \mathrm{mL}$ using inhibition zone diameter in $\mathrm{mm}$ as a criterion for the antimicrobial activity, and the results are shown in (Table 1). Based on the results, the newly synthesized compounds tested displayed variable invitro antimicrobial activities under these screening conditions. Interestingly, the tested compounds exhibited significant antifungal activities against the filamentous fungus (Aspergillus fumigatus) and unicellular yeast (Candida albicans). The highest antifungal activity was detected for compound $\mathbf{3 b}$ followed by 3a, 3e, 3d, 3c, respectively. However, compound $3 \mathbf{e}$ exhibited the highest activity against Gram-positive bacteria, Staphylococcus aureus as compared with the standard antibiotic, Ampicillin, 
followed by $\mathbf{3 b}, \mathbf{3 a}, \mathbf{8 a}$ and $\mathbf{3 d}$, respectively. On the other hand, compound $\mathbf{3 b}$ exhibited the highest activity against Gram-positive bacteria, Bacillus subtilis followed by 3a, 3e, 3d and 6a, respectively. Moreover, the tested Gram-negative bacteria; Proteus vulgaris was highly susceptible to compound $\mathbf{3 e}$ followed by, 3d, 8a, $\mathbf{3 b}, \mathbf{3 a}, \mathbf{6 a}$ and $\mathbf{6 b}$ as compared with the standard antibiotic, Gentamycin. The order of activity against Escherichia coli was $\mathbf{3 e}>\mathbf{3 b}>\mathbf{3 a}>\mathbf{3 d}>\mathbf{6 a}>\mathbf{8 a}>\mathbf{3 c}>\mathbf{6 c}$ $>6 \mathrm{~b}>8 \mathrm{~b}$.

Table 1. In-vitro antimicrobial activities of the synthesized compounds tested at $10 \mathrm{mg} / \mathrm{mL}$ by well diffusion agar assay and expressed as inhibition zone diameter $(\mathrm{mm})$ in the form of mean \pm standard deviation.

\begin{tabular}{|c|c|c|c|c|c|c|}
\hline \multirow[b]{2}{*}{ Compound } & \multicolumn{2}{|c|}{ Fungi } & \multicolumn{2}{|c|}{ Gram positive bacteria } & \multicolumn{2}{|c|}{ Gram negative bacteria } \\
\hline & $\begin{array}{l}\text { C. albicans } \\
\text { ATCC } \\
\mathbf{1 0 2 3 1}\end{array}$ & $\begin{array}{l}\text { A. fumigatus } \\
\text { RCMB } \\
002568\end{array}$ & $\begin{array}{c}\text { S. Aureus } \\
\text { RCMB } 010012\end{array}$ & $\begin{array}{l}\text { B. subtilis } \\
\text { NRRL } \\
\text { B-543 }\end{array}$ & $\begin{array}{c}\text { Proteus vulgaris } \\
\text { ATCC } 13315\end{array}$ & $\begin{array}{l}\text { E. coli } \\
\text { ATCC } \\
25955\end{array}$ \\
\hline $\mathbf{3 a}$ & $33.5 \pm 1.6$ & $29 \pm 0.7$ & $20.3 \pm 0.5$ & $18.4 \pm 0.8$ & $15.8 \pm 1.4$ & $21.2 \pm 0.4$ \\
\hline $3 \mathbf{b}$ & $34.1 \pm 1.7$ & $30.7 \pm 1.5$ & $21.6 \pm 1.3$ & $20.2 \pm 0.6$ & $16.1 \pm 0.7$ & $22.6 \pm 0.8$ \\
\hline $3 c$ & $13 \pm 0.7$ & $20.3 \pm 1.1$ & $15.8 \pm 0.5$ & $8.1 \pm 0.2$ & $12.4 \pm 0.6$ & $13.1 \pm 0.4$ \\
\hline 3d & $14.2 \pm 0.6$ & $26 \pm 1.2$ & $17.1 \pm 0.8$ & $15.4 \pm 0.7$ & $17.1 \pm 0.8$ & $20.4 \pm 1.6$ \\
\hline $3 e$ & $26.1 \pm 1.2$ & $27.4 \pm 0.8$ & $22.3 \pm 1.4$ & $16 \pm 0.8$ & $18.9 \pm 1.6$ & $23.4 \pm 0.9$ \\
\hline $\mathbf{6 a}$ & $17.1 \pm 0.7$ & $15.7 \pm 0.9$ & $16.3 \pm 1.1$ & $15.2 \pm 1.4$ & $14.7 \pm 1.3$ & $17.5 \pm 1.2$ \\
\hline $6 \mathbf{b}$ & 0 & 0 & $13.4 \pm 0.9$ & $9.2 \pm 0.5$ & $14.5 \pm 0.8$ & $10.1 \pm 0.7$ \\
\hline $6 c$ & $11.4 \pm 0.9$ & 0 & $13.3 \pm 0.8$ & $10.2 \pm 0.6$ & $13.5 \pm 0.7$ & $11.6 \pm 0.5$ \\
\hline $\mathbf{8 a}$ & $14.5 \pm 1.1$ & $16 \pm 0.8$ & $18.2 \pm 0.9$ & $14 \pm 0.6$ & $16.4 \pm 0.8$ & $15.1 \pm 1.3$ \\
\hline $8 b$ & $14.6 \pm 0.8$ & $12.9 \pm 0.3$ & $10.2 \pm 0.4$ & $11.5 \pm 0.7$ & $10.9 \pm 0.4$ & $9.7 \pm 0.5$ \\
\hline $11 \mathbf{a}$ & 0 & 0 & 0 & $8.6 \pm 0.8$ & 0 & $\mathbf{0}$ \\
\hline $11 b$ & 0 & 0 & 0 & 0 & 0 & $\mathbf{0}$ \\
\hline 11c & 0 & 0 & $9.8 \pm 0.6$ & 0 & 0 & $\mathbf{0}$ \\
\hline 11d & 0 & 0 & $8.7 \pm 0.5$ & 0 & 0 & $\mathbf{0}$ \\
\hline 11e & 0 & 0 & 0 & $10.2 \pm 0.6$ & 0 & $\mathbf{0}$ \\
\hline Amphotricin B & $25.7 \pm 1.3$ & $24.8 \pm 1.4$ & - & - & - & - \\
\hline Ampicillin & - & - & $27.8 \pm 0.6$ & $26.4 \pm 0.7$ & - & - \\
\hline Gentamycin & - & - & - & - & $25.7 \pm 0.9$ & $29.6 \pm 1.3$ \\
\hline
\end{tabular}

Amphotericin B, ampicillin and gentamycin were used as standard drugs against the tested fungi, Grampositive and Gram-negative bacteria, respectively.

The antimicrobial activities of the synthesized compounds were also tested to determine the minimum inhibitory concentration as shown in Table 2. Moreover, compound 3b showed the highest activity (MIC values ranged from 4.9 to $312.5 \mu \mathrm{g} / \mathrm{ml}$ ), followed by 3a (MIC 4.9-625 $\mu \mathrm{g} / \mathrm{ml}$ ), 3e (MIC 9.8-625 $\mu \mathrm{g} / \mathrm{ml})$, and 3d (MIC 9.8-625 $\mu \mathrm{g} / \mathrm{ml})$. 
Table 2. The antibacterial activities of the synthesized compounds expressed as minimum inhibitory concentration $(\mu \mathrm{g} / \mathrm{ml})$.

\begin{tabular}{|c|c|c|c|c|c|c|}
\hline \multirow[b]{2}{*}{ Compound } & \multicolumn{2}{|c|}{ Fungi } & \multicolumn{2}{|c|}{ Gram positive bacteria } & \multicolumn{2}{|c|}{ Gram negative bacteria } \\
\hline & $\begin{array}{l}\text { C. albicans } \\
\text { ATCC } \\
10231\end{array}$ & $\begin{array}{l}\text { A. fumigatus } \\
\text { RCMB } \\
002568\end{array}$ & $\begin{array}{l}\text { S. aureus } \\
\text { RCMB } \\
010012\end{array}$ & $\begin{array}{l}\text { B. subtilis } \\
\text { NRRL B- } \\
543\end{array}$ & $\begin{array}{c}\text { Proteus } \\
\text { vulgaris ATCC } \\
\mathbf{1 3 3 1 5}\end{array}$ & $\begin{array}{l}\text { E. coli } \\
\text { ATCC } \\
25955\end{array}$ \\
\hline 3a & 4.9 & 4.9 & 39 & 156 & 625 & 39 \\
\hline 3b & 4.9 & 4.9 & 156 & 156 & 312.5 & 39 \\
\hline $3 c$ & 1250 & 156 & 625 & 5000 & 1250 & 625 \\
\hline $3 d$ & 625 & 9.8 & 312.5 & 625 & 312.5 & 156 \\
\hline $3 e$ & 9.8 & 9.8 & 39 & 625 & 156 & 39 \\
\hline 6a & 312.5 & 625 & 625 & 625 & 625 & 312.5 \\
\hline $6 b$ & $\mathrm{NA}^{*}$ & NA & 625 & 5000 & 625 & 2500 \\
\hline $6 c$ & 1250 & NA & 625 & 2500 & 625 & 2500 \\
\hline $8 \mathbf{a}$ & 625 & 625 & 312.5 & 625 & 625 & 625 \\
\hline $8 b$ & 625 & 1250 & 2500 & 1250 & 2500 & 2500 \\
\hline Amphotricin B & 9.8 & 2.44 & - & - & - & - \\
\hline Ampicillin & - & - & 1.22 & 0.6 & 9.76 & 9.76 \\
\hline Gentamycin & - & - & 9.76 & 4.88 & 0.6 & 1.22 \\
\hline
\end{tabular}

* NA: No activity

\section{Conclusion}

A series of novel 4-((4-hydroxynaphthalen-1yl)diazenyl) benzenesulfonamides, naphtho[1,2$b]$ furans, benzo[ $h]$ chromenes and 4-(4-amino-3,5dicyano-6-imino-5,6-dihydropyridazin-1(4H)-

yl)benzenesulfonamides were synthesized to evaluate their antimicrobial biological activity with the hope of discovering new structure leads serving as antimicrobial agents.

\section{Experimental}

All analyses were done at the Microanalytical Center, Cairo University, Cairo (Egypt). Melting points (uncorrected) were determined in open capillaries on a Gallenkamp melting point apparatus (Sanyo Gallenkamp, Southborough, UK). IR spectra $(\mathrm{KBr}$ discs) were recorded using a Shimadzu FT-IR 8400 S spectrophotometer (Shimadzu, Kyoto, Japan), Infrared (IR) Spectra were recorded as KBr disks. NMR Spectra were recorded on a Bruker spectrophotometer (Bruker,
Karlsruhe, Germany). ${ }^{1} \mathrm{H}$ spectrum was run at $400 \mathrm{MHz}$ in deuterated dimethylsulfoxide (DMSO- $d_{6}$ ). Chemical shifts are expresses in values (ppm) relative to TMS as an internal standard. Mass spectral data were given by a GCMS-QP1000 EX-spectrometer (Shimadzu, Kyoto, Japan) at $70 \mathrm{eV}$. All reagents used were of the Analytical grade. Compounds $\alpha$-cyanocinnamonitriles $7^{41}$ and 2amino-1,1,3-tricyanopropene $9^{42}$ have been synthesized as previously reported.

General Procedure for Synthesis of 4-((4hydroxynaphthalen-1-yl)diazenyl)benzenesulfonamides 3a-e:

Sulfonamide (0.01 mole) was suspended in water $(50 \mathrm{ml})$. Hydrochloric acid $(10 \mathrm{ml}, 36 \%)$ was added dropwise to this well stirred. The mixture was gradually heated up to $70{ }^{\circ} \mathrm{C}$ till clear solution obtained. The solution was cooled to $0-5^{\circ} \mathrm{C}$ in an ice bath. A solution of $\mathrm{NaNO}_{2}(0.5 \mathrm{mg})$ in water $(5 \mathrm{ml})$ previously cooled to $0{ }^{\circ} \mathrm{C}$, was then added over a period 5 minutes with 
stirring. 1-naphthol (0.01 mole) was dissolved in $10 \%$ $\mathrm{NaOH}(10 \mathrm{ml})$ and then put ice to cool to $5{ }^{\circ} \mathrm{C}$. Then, diazonium salt solution was added occasionally stirring very slowly to the 1-naphthol solution. The reaction mixture was left to complete for $15 \mathrm{~min}$ and occasional stirring; then the formed precipitate was filtered and dried in air and then recrystallized from proper solvent to give $\mathbf{3}$.

\section{4-((4-hydroxynaphthalen-1-yl)diazenyl)benzene- sulfonamide $3 a$.}

Brown crystals (ethanol), Yield:85\%, m.p.254-256 ${ }^{\circ} \mathrm{C}$; $\mathrm{IR}\left(\mathrm{KBr}, \mathrm{cm}^{-1}\right)$ : $3357(\mathrm{OH}), 3300,3249\left(\mathrm{NH}_{2}\right), 1622$ $(\mathrm{C}=\mathrm{C}), 1594(\mathrm{~N}=\mathrm{N}), 1356,1147(\mathrm{~S}=\mathrm{O})$;

${ }^{1} \mathrm{HNMR}$ (DMSO- $\left.d_{6}, \mathrm{ppm}\right):$ 7.24, 7.72, 7.98, 8.16(4d, $4 \mathrm{H}), 7.55,7.62(2 \mathrm{~m}, 2 \mathrm{H}), 7.88,8.26(2 \mathrm{~d}, 4 \mathrm{H}), 7.36(\mathrm{~s}$, $2 \mathrm{H}, \mathrm{NH}_{2}$, exchangeable with $\left.\mathrm{D}_{2} \mathrm{O}\right), 9.45(\mathrm{~s}, 1 \mathrm{H}, \mathrm{OH}$, exchangeable with $\mathrm{D}_{2} \mathrm{O}$ );

Anal. Calcd. for $\mathrm{C}_{16} \mathrm{H}_{13} \mathrm{~N}_{3} \mathrm{O}_{3} \mathrm{~S}: \mathrm{C}, 58.70 ; \mathrm{H}, 4.00 ; \mathrm{N}$, 12.84; S, 9.80. Found: C, 58.52; H, 3.89; N, 12.58; S, 9.65 .

\section{N-carbamimidoyl-4-((4-hydroxynaphthalen-1- yl)diazenyl)benzenesulfonamide $3 b$.}

Brown crystals (ethanol), Yield:83\%, m.p.246-248 ${ }^{\circ} \mathrm{C}$; $\mathrm{IR}(\mathrm{KBr}, \mathrm{cm}-1)$ : $3441(\mathrm{OH}), 3329\left(\mathrm{NH}_{2}\right), 3272,3223$ $(2 \mathrm{NH}), 1633(\mathrm{C}=\mathrm{C}), 1596(\mathrm{~N}=\mathrm{N}), 1355,1165(\mathrm{~S}=\mathrm{O})$; ${ }^{1} \mathrm{HNMR}$ (DMSO- $\left.d_{6}, \mathrm{ppm}\right):$ 7.28, $7.72,8.14(3 \mathrm{~d}, 3 \mathrm{H})$, 7.49-7.57(2m, 2H), 7.82, $788(2 \mathrm{~d}, 4 \mathrm{H}), 6.75(\mathrm{~s}, 2 \mathrm{H}$, $\mathrm{NH}_{2}$, exchangeable with $\left.\mathrm{D}_{2} \mathrm{O}\right), 7.61,7.80(2 \mathrm{~s}, 2 \mathrm{H}, 2 \mathrm{NH}$, exchangeable with $\left.\mathrm{D}_{2} \mathrm{O}\right), 10.03(\mathrm{~s}, 1 \mathrm{H}, \mathrm{OH}$, exchangeable with $\left.\mathrm{D}_{2} \mathrm{O}\right)$; Anal. Calcd. for $\mathrm{C}_{17} \mathrm{H}_{15} \mathrm{~N}_{5} \mathrm{O}_{3} \mathrm{~S}$ : C, 55.27; H, 4.09; N, 18.96; S, 8.68. Found: C, 55.12; H, 3.95; N, 18.76; S, 8.42.

4-((4-hydroxynaphthalen-1-yl)diazenyl)-N-(thiazol-2yl)benzenesulfonamide $3 c$.

Brown crystals (ethanol), Yield: 86\%, m.p.202-204 ${ }^{\circ} \mathrm{C}$; IR(KBr, cm-1): $3415(\mathrm{OH}), 3274(\mathrm{NH}), 1627$ (C=C), $1594(\mathrm{~N}=\mathrm{N}), 1317,1138(\mathrm{~S}=\mathrm{O})$;

${ }^{1} \mathrm{HNMR}$ (DMSO- $\left.d_{6}, \mathrm{ppm}\right): 7.30,7.75,8.13,8.15(4 \mathrm{~d}$, $4 \mathrm{H}), 7.58,7.71(2 \mathrm{~m}, 2 \mathrm{H}), 7.86,7.92(2 \mathrm{~d}, 4 \mathrm{H}), 6.73,7.26$ (2d, 2H, H-thiazole), 12.74 (br, H, NH, exchangeable with $\left.\mathrm{D}_{2} \mathrm{O}\right), 10.05\left(\mathrm{~s}, 1 \mathrm{H}, \mathrm{OH}\right.$, exchangeable with $\left.\mathrm{D}_{2} \mathrm{O}\right)$; MS: $410\left(\mathrm{M}^{+} ; 42.83 \%\right), 393$ (3.17), 382 (28.92\%), 346 (62.87\%), 247 (17.45\%), 219 (4.18\%), 92 (93.25\%), 76(19.77\%), 65 (100\%).

Anal. Calcd. for $\mathrm{C}_{19} \mathrm{H}_{14} \mathrm{~N}_{4} \mathrm{O}_{3} \mathrm{~S}_{2}$ : C, 55.60; H, 3.44; N, 13.65; S, 15.62. Found: C, 55.46; H, 3.28; N, 13.47; S, 15,42 .

\section{4-((4-hydroxynaphthalen-1-yl)diazenyl)-N-(5- methylisoxazol-3-yl)benzenesulfonamide $3 d$.}

Brown crystals (ethanol), Yield: 80\%, m.p.242-244 ${ }^{\circ} \mathrm{C}$; IR(KBr, cm-1): 3448 (OH), 3107 (NH), 2924 (CH- aliph.), $1632(\mathrm{C}=\mathrm{C}), 1595(\mathrm{~N}=\mathrm{N}), 1327,1139(\mathrm{~S}=\mathrm{O})$; ${ }^{1} \mathrm{HNMR}$ (DMSO-d $\left.d_{6}, \mathrm{ppm}\right): 2.36\left(\mathrm{~s}, 3 \mathrm{H}, \mathrm{CH}_{3}\right), 6.25$ (s, 1H, H-oxazole), 7.25, 7.68, 8.04, 8.12(4d, 4H), 7.51, $7.63(2 \mathrm{~m}, 2 \mathrm{H}), 7.88,7.97(2 \mathrm{~d}, 4 \mathrm{H}), 11.26(\mathrm{~s}, \mathrm{H}, \mathrm{NH}$, exchangeable with $\left.\mathrm{D}_{2} \mathrm{O}\right), 10.02(\mathrm{~s}, 1 \mathrm{H}, \mathrm{OH}$, exchangeable with $\mathrm{D}_{2} \mathrm{O}$ );

Anal. Calcd. for $\mathrm{C}_{20} \mathrm{H}_{16} \mathrm{~N}_{4} \mathrm{O}_{4} \mathrm{~S}: \mathrm{C}, 58.81 ; \mathrm{H}, 3.95 ; \mathrm{N}$, 13.72; S, 7.85. Found: C, 58.64; H, 3.79; N, 13.54; S, 7.68 .

4-((4-hydroxynaphthalen-1-yl)diazenyl)- $\mathrm{N}$ (pyrimidin-2-yl)benzenesulfonamide $3 e$.

Dark brown crystals (ethanol), Yield:7 8\%, m.p. 132$134{ }^{\circ} \mathrm{C}$;

IR(KBr, cm-1): $3377(\mathrm{OH}), 3225(\mathrm{NH}), 1625$ (C=C), $1581(\mathrm{~N}=\mathrm{N}), 1316,1155(\mathrm{~S}=\mathrm{O})$;

${ }^{1} \mathrm{HNMR}$ (DMSO-d, $\left.\mathrm{ppm}\right): 6.99,8.36(\mathrm{~m}, \mathrm{~d}, 3 \mathrm{H}, \mathrm{H}-$ pyrimidine), 7.23, 7.69, 8.06, $8.15(4 \mathrm{~d}, 4 \mathrm{H}), 7.52,7.60$ $(2 \mathrm{~m}, 2 \mathrm{H}), 7.91,7.98(2 \mathrm{~d}, 4 \mathrm{H}), 11.30(\mathrm{~s}, \mathrm{H}, \mathrm{NH}$, exchangeable with $\left.\mathrm{D}_{2} \mathrm{O}\right), 9.98(\mathrm{~s}, 1 \mathrm{H}, \mathrm{OH}$, exchangeable with $\mathrm{D}_{2} \mathrm{O}$ );

Anal. Calcd. for $\mathrm{C}_{20} \mathrm{H}_{15} \mathrm{~N}_{5} \mathrm{O}_{3} \mathrm{~S}$ : C, 59.25; H, 3.73; N, 17.27; S, 7.91. Found: C, 58.94; H, 3.64; N, 17.13; S, 7.64 .

\section{General Procedure for Synthesis of naphtho[1,2- b]furans 6 a-c.}

To a mixture of compound 3 (0.01 mole) and cinnamic acid 4 (0.01 mole) in DMF $(10 \mathrm{ml})$, a few drops piperidine was added. The reaction mixture was refluxed for $2 \mathrm{~h}$. After cooling, the precipitate was filtered and recrystallized from proper solvent to give 6.

\section{4-((3-phenylnaphtho[1,2-b]furan-5-yl)diazenyl)- benzenesulfonamide $6 a$.}

Brown crystals (ethanol), Yield: 79\%, m.p.180-182 ${ }^{\circ} \mathrm{C}$; $\mathrm{IR}(\mathrm{KBr}, \mathrm{cm}-1): 3266\left(\mathrm{NH}_{2}\right), 1624(\mathrm{C}=\mathrm{C}), 1594(\mathrm{~N}=\mathrm{N})$, $1316,1155(\mathrm{~S}=\mathrm{O})$;

${ }^{1} \mathrm{HNMR}$ (DMSO- $\left.d_{6}, \mathrm{ppm}\right):$ 7.28-7.55 (m, d, 5H, Ph-H), $8.18(\mathrm{~s}, 1 \mathrm{H}$, furan-H), 8.32(s, 1H), 8.20, 8.52(2d, $2 \mathrm{H})$, 7.42, $7.56(2 \mathrm{~m}, 2 \mathrm{H}), 7.90,8.06(2 \mathrm{~d}, 4 \mathrm{H}), 7.30(\mathrm{~s}, 2 \mathrm{H}$, $\mathrm{NH}_{2}$, exchangeable with $\mathrm{D}_{2} \mathrm{O}$ );

Anal. Calcd. for $\mathrm{C}_{24} \mathrm{H}_{17} \mathrm{~N}_{3} \mathrm{O}_{3} \mathrm{~S}$ : C, 67.43; H, 4.01; N, 11.23; S, 7.50. Found: C, 67.28; H, 3.87; N, 11.14; S, 7.38 .

N-carbamimidoyl-4-((3-phenylnaphtho[1,2-b]furan5-yl)diazenyl)benzenesulfonamide $6 b$.

Dark brown crystals (ethanol), Yield: $82 \%$, m.p.205$207^{\circ} \mathrm{C}$;

IR(KBr, cm-1): $3440\left(\mathrm{NH}_{2}\right), 3328,3271(2 \mathrm{NH}), 1632$ $(\mathrm{C}=\mathrm{C}), 1595(\mathrm{~N}=\mathrm{N}), 1307,1131(\mathrm{~S}=\mathrm{O})$;

${ }^{1} \mathrm{HNMR}$ (DMSO-d 6 , ppm): 7.32-7.58 (m, d, 5H, Ph-H), $8.17(\mathrm{~s}, 1 \mathrm{H}$, furan-H), 8.32(s, 1H), 8.17, $8.43(2 \mathrm{~d}, 2 \mathrm{H})$, 7.60, 7.67 (2m, 2H), 7.88, 7.96(2d, 4H), 6.74(s, 2H, 
$\mathrm{NH}_{2}$, exchangeable with $\left.\mathrm{D}_{2} \mathrm{O}\right), 7.75,8.08(2 \mathrm{~s}, 2 \mathrm{H}, 2 \mathrm{NH}$, exchangeable with $\mathrm{D}_{2} \mathrm{O}$ ).

MS: $469\left(\mathrm{M}^{+} ; 12.15 \%\right), 453$ (8.95\%), 426 (10.10\%), 398 (6.67\%), 392(6.67\%), 362 (14.58\%), 198 (11.76 $\%), 135(8.31 \%), 57(81.71 \%), 55(100 \%)$.

Anal. Calcd. for $\mathrm{C}_{25} \mathrm{H}_{19} \mathrm{~N}_{5} \mathrm{O}_{3} \mathrm{~S}$ : C, 63.95; H, 4.08; N, 10.22; S, 6.83. Found: C, 63.78; H, 3.97; N, 10.10; S, 6.65 .

\section{4-((3-phenylnaphtho[1,2-b]furan-5-yl)diazenyl)-N- (thiazol-2-yl)benzenesulfonamide $6 c$.}

Dark brown crystals (ethanol), Yield: 84\%, m.p.193$195^{\circ} \mathrm{C}$;

IR(KBr, cm-1): 3247 (NH), $1628(\mathrm{C}=\mathrm{C}), 1593(\mathrm{~N}=\mathrm{N})$, 1311, $1137(\mathrm{~S}=\mathrm{O})$;

${ }^{1}$ HNMR (DMSO-d 6 , ppm): 6.73, 7.19 (2d, 2H, thiazole$\mathrm{H}), 7.35-7.58$ (m, d, 5H, Ph-H), $8.12(\mathrm{~s}, 1 \mathrm{H}$, furan-H), 8.26( s, 1H), 8.14, 8.44 (2d, 2H), $7.43,7.59$ (2m, 2H), 7.93, $8.02(2 \mathrm{~d}, 4 \mathrm{H}), 12.64(\mathrm{~s}, \mathrm{H}, \mathrm{NH}$, exchangeable with $\left.\mathrm{D}_{2} \mathrm{O}\right)$;

MS: $510\left(\mathrm{M}^{+} ; 7.64 \%\right), 494$ (4.64\%), 482 (6.39\%), 446 $(4.76 \%), 433(4.14 \%), 319(3.38 \%), 267(3.45 \%), 241$ (7.58\%), $239(3.29 \%), 226(4.26 \%), 209(4.07 \%), 177$ $(4.57 \%), 149(4.76 \%), 84(10.24 \%), 58$ (27.69), 57 (100\%), 56 (32.02\%), 52 (7.89\%). Anal. Calcd. for $\mathrm{C}_{27} \mathrm{H}_{18} \mathrm{~N}_{4} \mathrm{O}_{3} \mathrm{~S}_{2}$ : C, 63.51; H, 3.55; N, 10.97; S, 12.56. Found: C, 63.38; H, 3.39; N, 10.75; S, 12.32.

\section{General Procedure for Synthesis of benzo[h]chromenes $8 a, b$.}

To a mixture of compound 3 (0.01 mole) and $\alpha$-cyanocinnamonitrile 7(0.01 mole) in DMF (10 ml), a few drops triethylamine was added. The reaction mixture was refluxed for $1 \mathrm{~h}$. After cooling, the precipitate was filtered and recrystallized from proper solvent to give $\mathbf{8}$.

4-((2-amino-3-cyano-4-phenyl-4H-benzo[h]chromen6-yl)diazenyl)benzenesulfonamide $8 a$.

Brown crystals (ethanol), Yield: $84 \%$, m.p. $90-92{ }^{\circ} \mathrm{C}$; IR(KBr, cm-1): 3355, $3270\left(2 \mathrm{NH}_{2}\right), 3054$ (CH-arom.), $2190(\mathrm{CN}), 1631(\mathrm{C}=\mathrm{C}), 1593(\mathrm{~N}=\mathrm{N}), 1357,1156$ $(\mathrm{S}=\mathrm{O}) ;{ }^{1} \mathrm{HNMR}\left(\mathrm{DMSO}-\mathrm{d}_{6}, \mathrm{ppm}\right): 4.90$ (s, 1H, 4Hpyran), 7.23-7.32 (m, d, 5H, Ph-H), 7.88(s, 1H), 8.12, $8.27(2 \mathrm{~d}, 2 \mathrm{H}), 7.49,7.56(2 \mathrm{~m}, 2 \mathrm{H}), 7.90,8.25(2 \mathrm{~d}, 4 \mathrm{H})$, 6.88, $7.21\left(2 \mathrm{~s}, 4 \mathrm{H}, 2 \mathrm{NH}_{2}\right.$, exchangeable with $\left.\mathrm{D}_{2} \mathrm{O}\right)$; MS: $481\left(\mathrm{M}^{+} ; 1.64 \%\right), 465$ (1.53\%), 455 (1.55\%), 452 (25.81\%), $401(2.42 \%), 404(4.62 \%), 373(12 \%), 327$ (8.13\%), 297 (100\%), (269 (39.18\%), 219 (1.11\%), 65 $(26.78 \%)$.

Anal. Calcd. for $\mathrm{C}_{26} \mathrm{H}_{19} \mathrm{~N}_{5} \mathrm{O}_{3} \mathrm{~S}$ : C, 64.85; H, 3.98; N, 14.54; S, 6.66. Found: C, 64.63; H, 3.74; N, 14.24; S, 6.39 .
4-((2-amino-3-cyano-4-(p-tolyl)-4H-benzo[h]chromen-6-yl)diazenyl)benzenesulfonamide $8 b$.

Brown crystals (ethanol), Yield: $87 \%$, m.p.135-137 ${ }^{\circ} \mathrm{C}$; IR(KBr, cm-1): 3424, 3385 (2NH 2$), 3034$ (CH-arom), 2870 (CH-aliph), $2186(\mathrm{CN}), 1631(\mathrm{C}=\mathrm{C}), 1594(\mathrm{~N}=\mathrm{N})$, $1358,1153(\mathrm{~S}=\mathrm{O})$;

${ }^{1} \mathrm{HNMR}$ (DMSO-d 6 , ppm): 2.35 (s, 3H, $\mathrm{CH}_{3}$ ), 4.82 (s, 1H, 4H-pyran), 7.13, 7.21 (2d, 4H, Ph-CH3), 7.87(s, $1 \mathrm{H}), 8.13,8.38(2 \mathrm{~d}, 2 \mathrm{H}), 7.51,7.54(2 \mathrm{~m}, 2 \mathrm{H}), 8.15$, $7.81(2 \mathrm{~d}, 4 \mathrm{H}), 6.89,7.23\left(2 \mathrm{~s}, 4 \mathrm{H}, 2 \mathrm{NH}_{2}\right.$, exchangeable with $\mathrm{D}_{2} \mathrm{O}$ );

Anal. Calcd. for $\mathrm{C}_{27} \mathrm{H}_{21} \mathrm{~N}_{5} \mathrm{O}_{3} \mathrm{~S}$ : C, 65.44; H, 4.27; N, 14.13; S, 6.47. Found: C, 65.38; H, 4.09; N, 13.95; S, 6.28 .

General Procedure for Synthesis of 4-(4-amino-3,5dicyano-6-iminopyridazin-1(6H)-yl)benzenesulfonamides 11a-e:

Sulfonamide (0.01 mole) was suspended in water $(50 \mathrm{ml})$. Hydrochloric acid $(10 \mathrm{ml}, 36 \%)$ was added drop wise to this well stirred. The mixture was gradually heated up to $70{ }^{\circ} \mathrm{C}$ till clear solution obtained. The solution was cooled to $0-5^{\circ} \mathrm{C}$ in an ice bath. A solution of $\mathrm{NaNO}_{2}(0.5 \mathrm{gm})$ in water $(5 \mathrm{ml})$ previously cooled to $0{ }^{\circ} \mathrm{C}$, was then added over a period 5 minutes with stirring. 2-Amino-1,1,3-tricyanopropene 9 (0.01 mole) was dissolved in ethanol $(10 \mathrm{ml})$ in the presence of sodium acetate (1 gram) and then put ice to cool to $5^{\circ} \mathrm{C}$. Then, diazonium salt solution was added occasionally stirring very slowly to the 2-Amino-1,1,3tricyanopropene solution. The reaction mixture was left to complete for $3 \mathrm{~h}$ and occasional stirring; then the formed precipitate was filtered and dried in air and then recrystallized from proper solvent to give $\mathbf{1 1}$.

\section{4-(4-amino-3,5-dicyano-6-iminopyridazin-1(6H)-} yl)benzenesulfonamide 11a:

Yellow crystals (ethanol), Yield: $88 \%$, m.p.158- $160{ }^{\circ} \mathrm{C}$; $\mathrm{IR}(\mathrm{KBr}, \mathrm{cm}-1)$ : 3378, $3312\left(2 \mathrm{NH}_{2}\right), 3211(\mathrm{NH}), 2219$, $2199(2 \mathrm{C} \equiv \mathrm{N}), 1620(\mathrm{C}=\mathrm{N}), 1326,1159(\mathrm{~S}=\mathrm{O})$;

${ }^{1}$ HNMR (DMSO-d 6 , ppm): 6.65, 7.20 (2br., 4H, 2NH ${ }_{2}$, exchangeable with $\left.\mathrm{D}_{2} \mathrm{O}\right), 7.80,8.21(2 \mathrm{~d}, 4 \mathrm{H}, \mathrm{AB}-$ system), 9.85(br., $1 \mathrm{H}, \mathrm{NH}$, exchangeable with $\mathrm{D}_{2} \mathrm{O}$ ).

Anal. Calcd. for $\mathrm{C}_{12} \mathrm{H}_{9} \mathrm{~N}_{7} \mathrm{O}_{2} \mathrm{~S}: \mathrm{C}, 45.71 ; \mathrm{H}, 2.88 ; \mathrm{N}$, 31.10; S, 10.17. Found: C, 45.56; H, 2.64; N, 30.94; S, 10.04 .

\section{4-(4-amino-3,5-dicyano-6-iminopyridazin-1(6H)-yl)- $\mathrm{N}$-carbamimidoylbenzenesulfon amide $11 \mathrm{~b}$}

Yellow crystals (ethanol), Yield: $82 \%$, m.p. $>300{ }^{\circ} \mathrm{C}$; $\mathrm{IR}(\mathrm{KBr}, \mathrm{cm}-1)$ : 3450-3211 (NH+NH$), 2220,2202$ $(2 \mathrm{C} \equiv \mathrm{N}), 1620(\mathrm{C}=\mathrm{N}), 1352,1138(\mathrm{~S}=\mathrm{O})$;

${ }^{1} \mathrm{HNMR}$ (DMSO-d 6 , ppm): 6.85, 7.10(2br., 4H, 2NH , exchangeable with $\left.\mathrm{D}_{2} \mathrm{O}\right), 7.63,7.95(2 \mathrm{~d}, 4 \mathrm{H}, \mathrm{AB}-$ 
system), 7.55, 8.52, 10.10(3br., 3H, 3NH, exchangeable with $\mathrm{D}_{2} \mathrm{O}$ ).

Anal. Calcd. for $\mathrm{C}_{13} \mathrm{H}_{11} \mathrm{~N}_{9} \mathrm{O}_{2} \mathrm{~S}: \mathrm{C}, 43.69 ; \mathrm{H}, 3.10 ; \mathrm{N}$, 35.28; S, 8.97. Found: C, 43.46; H, 2.92; N, 35.13; S, 8.87 .

\section{4-(4-amino-3,5-dicyano-6-iminopyridazin-1(6H)-yl)- $\mathrm{N}$-(thiazol-2-yl)benzenesulfon amide 11c}

Yellow crystals (ethanol), Yield: $85 \%$, m.p. $>300{ }^{\circ} \mathrm{C}$; $\mathrm{IR}(\mathrm{KBr}, \mathrm{cm}-1): 3430\left(\mathrm{NH}_{2}\right), 3320,3214(2 \mathrm{NH}), 2216$, $2201(2 \mathrm{C} \equiv \mathrm{N}), 1622(\mathrm{C}=\mathrm{N}), 1330,1147(\mathrm{~S}=\mathrm{O})$;

${ }^{1} \mathrm{HNMR}$ (DMSO-d 6 , ppm): 6.84, 7.77 (2d, 2H, thiazole), 7.70, $7.95\left(2 \mathrm{~d}, 4 \mathrm{H}, \mathrm{AB}\right.$-system), $6.70\left(\mathrm{~s}, 2 \mathrm{H}, \mathrm{NH}_{2}\right.$, exchangeable with $\mathrm{D}_{2} \mathrm{O}$ ), 9.85, 12.16 (2br., $2 \mathrm{H}, 2 \mathrm{NH}$, exchangeable with $\mathrm{D}_{2} \mathrm{O}$ ).

Anal. Calcd. for $\mathrm{C}_{15} \mathrm{H}_{10} \mathrm{~N}_{8} \mathrm{O}_{2} \mathrm{~S}_{2}: \mathrm{C}, 45.22 ; \mathrm{H}, 2.53 ; \mathrm{N}$, 28.12; S, 16.10. Found: C, 45.22; H, 2.53; N, 28.12; S, 16.10 .

\section{4-(4-amino-3,5-dicyano-6-iminopyridazin-1 $(6 H)$-yl)- $\mathrm{N}$-(5-methylisoxazol-3-yl)benzenesulfonamide 11d:}

Yellow crystals (ethanol), Yield: $80 \%$, m.p. $>300{ }^{\circ} \mathrm{C}$; IR(KBr, cm-1): $3311\left(\mathrm{NH}_{2}\right), 3225,3192(2 \mathrm{NH}), 2217$, $2199(2 \mathrm{C} \equiv \mathrm{N}), 1618(\mathrm{C}=\mathrm{N}), 1330,1159(\mathrm{~S}=\mathrm{O})$;

${ }^{1} \mathrm{HNMR}$ (DMSO-d $\left.6, \mathrm{ppm}\right): 2.35$ (s, 3H, $\left.\mathrm{CH}_{3}\right), 6.15$ (s, $1 \mathrm{H}$, oxazole-H), 7.72, 8.12(2d, 4H, AB-system), 7.52(s, $2 \mathrm{H}, \mathrm{NH}_{2}$, exchangeable with $\left.\mathrm{D}_{2} \mathrm{O}\right), 9.76,11.18(2 \mathrm{~s}, 2 \mathrm{H}$, $2 \mathrm{NH}$, exchangeable with $\mathrm{D}_{2} \mathrm{O}$ );

Anal. Calcd. for $\mathrm{C}_{16} \mathrm{H}_{12} \mathrm{~N}_{8} \mathrm{O}_{3} \mathrm{~S}: \mathrm{C}, 48.48 ; \mathrm{H}, 3.05 ; \mathrm{N}$, 28.27; S, 8.09. Found: C, 48.26; H, 2.97; N, 28.12; S, 7.95 .

4-(4-amino-3,5-dicyano-6-iminopyridazin-1(6H)-yl)$\mathrm{N}$-(pyrimidin-2-yl)benzenesulfonamide $11 e$

Yellow crystals (ethanol), Yield: $88 \%$, m.p. $>300{ }^{\circ} \mathrm{C}$; $\mathrm{IR}(\mathrm{KBr}, \mathrm{cm}-1): 3434\left(\mathrm{NH}_{2}\right), 3228,3213(2 \mathrm{NH}), 2216$, $2201(2 \mathrm{C} \equiv \mathrm{N}), 1621(\mathrm{C}=\mathrm{N}), 1339,1155(\mathrm{~S}=\mathrm{O})$;

${ }^{1} \mathrm{HNMR}$ (DMSO-d $\left.\mathrm{d}_{6}, \mathrm{ppm}\right): 6.98,8.42(\mathrm{~m}, \mathrm{~d}, 3 \mathrm{H}, \mathrm{H}-$ pyrimidine), 7.68, 8.15 (2d, 4H, AB-system), 7.90(s, $2 \mathrm{H}, \mathrm{NH}_{2}$ exchangeable with $\left.\mathrm{D}_{2} \mathrm{O}\right), 9.65,11.26(2 \mathrm{~s}, 2 \mathrm{H}$, $2 \mathrm{NH}$, exchangeable with $\mathrm{D}_{2} \mathrm{O}$ );

Anal. Calcd. for $\mathrm{C}_{16} \mathrm{H}_{11} \mathrm{~N}_{9} \mathrm{O}_{2} \mathrm{~S}: \mathrm{C}, 48.85 ; \mathrm{H}, 2.82 ; \mathrm{N}$, 32.05; S, 8.15. Found: C, 48.64; H, 2.59; N, 31.97; S, 7.98 .

\section{Biological evaluation}

All microbial strains were provided from the culture collection of the Regional Center for Mycology and Biotechnology (RCMB), Al-Azhar University, Cairo, Egypt. The antimicrobial activity was investigated on a dozen of newly synthesized compounds in order to increase the selectivity of these derivatives towards test microorganisms using the agar diffusion method using Mueller-Hinton agar medium for bacteria and Sabouraud's agar medium for fungi ${ }^{43,44}$. Briefly, $100 \mu 1$ of the test bacteria/fungi were grown in $10 \mathrm{~mL}$ of fresh media until they reached a count of approximately $10^{8}$ cells $/ \mathrm{ml}$ for bacteria or $10^{5}$ cells $/ \mathrm{mL}$ for fungi. All the newly synthesized compounds were weighed and dissolved in dimethyl sulfoxide to prepare extract stock solution.

One hundred $\mu \mathrm{L}$ of each sample at $5 \mathrm{mg} / \mathrm{mL}$ was added to each well $(10 \mathrm{~mm}$ diameter holes cut in the agar gel). The plates were incubated for $24-48 \mathrm{~h}$ at $37{ }^{\circ} \mathrm{C}$ (for bacteria and yeast) and $48 \mathrm{~h}$ at $28^{\circ} \mathrm{C}$ (for filamentous fungi). After incubation, the microorganism's growth was observed. Ampicillin and Gentamycin were used as standard antibacterial drugs while amphotericin B was used as a standard antifungal drug. The resulting inhibition zone diameters were measured in millimeters and used as a criterion for the antimicrobial activity. If an organism is placed on the agar, it will not grow in the area around the well if it is susceptible to the chemical. This area of no growth around the disc is known as a Zone of inhibition. The size of the clear zone is proportional to the inhibitory action of the compound under investigation. Solvent controls (DMSO) were included in every experiment as negative controls. DMSO was used for dissolving the tested compounds and showed no inhibition zones, confirming that it does not influence the growth of the tested microorganisms. The active compounds were further investigated to determine their antimicrobial activity expressed regarding minimum inhibitory concentration (MIC) using the modified agar well diffusion method that mentioned above. Different concentrations of each active compound were tested and compared with standard drugs. The MIC was then determined as the lowest concentration inhibiting the growth of the organism after $24-48 \mathrm{~h}^{43,45}$.

\section{Conflict of interest}

The authors declare that they have no conflict of interest.

\section{References}

1- S. Omidi, V. Khojasteh, A. Kakanejadifard, M. Ghasemian, and F. Azarbani, Synthesis, characterization, spectroscopy and biological activity of 4-((3-formyl-4-hydroxyphenyl) azo)-1alkylpyridinium salts, J. Chem. Sci., 2018, 130, 114.

2- $\quad$ R. Rahman, A. Gul, and Z. Akhter, 4, 4'-Bis [4-(4'-hydroxyphenyl) phenylazo] diphenyl Ether, Molbank, 2016, 2016, M914.

3- E. Merino, Synthesis of azobenzenes: the coloured pieces of molecular materials, Chem. Soc. Rev., 2011, 40, 3835-3853.

4- H. Zollinger, Color Chemistry-Synthesis, 
Properties and Application of Organic Dyes and Pigments, VCH Publ. New York, 1987, 85-87.

5- P. F. Gordon, Organic Chemistry in Color, Springer: New York, NY, USA, 1987, 95-105.

6- D. Stevenson, Tartrazine, Azo, and Non-Azo Dyes. Food Allergy, 2008, 377.

7- X. Cheng, Q. Li, C. Li, J. Qin, and Z. Li, Azobenzene-Based Colorimetric Chemosensors for Rapid Naked-Eye Detection of Mercury (II), Chem. Eur. J., 2011, 17, 7276-7281.

8- W. J. Sandborn, Rational selection of oral 5aminosalicylate formulations and prodrugs for the treatment of ulcerative colitis. Nature Publishing Group, 2002.

9- K. Morihiro, O. Hasegawa, S. Mori, S. Tsunoda, and S. Obika, C5-azobenzene-functionalized locked nucleic acid uridine: isomerization properties, hybridization ability, and enzymatic stability, Org. Biomol. Chem., 2015, 13, 5209-5214.

10- L. Fang, S. Chen, X. Guo, Y. Zhang, and H. Zhang, Azobenzene-containing molecularly imprinted polymer microspheres with photo-and thermoresponsive template binding properties in pure aqueous media by atom transfer radical polymerization, Langmuir, 2012, 28, 9767-9777.

11- Y. Wang et al., Block copolymer aggregates with photo-responsive switches: towards a controllable supramolecular container, Polymer (Guildf)., 2009, 50, 4821-4828.

12- A. Natansohn and P. Rochon, Photoinduced motions in azo-containing polymers, Chem. Rev., 2002, 102, 4139-4176.

13- K. Itoga, M. Yamato, J. Kobayashi, A. Kikuchi, and T. Okano, Cell micropatterning using photopolymerization with a liquid crystal device commercial projector, Biomaterials, 2004, 25, 2047-2053.

14- T. Jaunet-Lahary, A. Chantzis, K. J. Chen, A. D. Laurent, and D. Jacquemin, Designing efficient azobenzene and azothiophene nonlinear optical photochromes, J. Phys. Chem. C, 2014, 118, 28831-28841.

15- W. R. Browne and B. L. Feringa, Making molecular machines work, Nat. Nanotechnol., 2006, 1, 25-35.

16- T. Lin et al., Red, green and blue reflections enabled in an optically tunable self-organized 3D cubic nanostructured thin film, Adv. Mater., 2013, 25, 5050-5054.

17- C. H. Lee, B. Bihari, R. Filler, and B. K. Mandal,

28- S. M. Monti, C. T. Supuran, and G. De Simone, Anticancer carbonic anhydrase inhibitors: a patent review (2008 - 2013), Expert Opin. Ther. Pat., 2013, 23, 737-749.

29- M. Kaya, E. Demir, and H. Bekci, Synthesis,
New azobenzene non-linear optical materials for eye and sensor protection, Opt. Mater. (Amst)., 2009, 32, 147-153.

18- O. Graydon, Photoisomerization: Molecular motors driven by light, Nat. Photonics, 2015, 9, 13.

19- L. Xia, A. Idhayadhulla, Y. R. Lee, Y.-J. Wee, and S. H. Kim, Anti-tyrosinase, antioxidant, and antibacterial activities of novel 5-hydroxy-4acetyl-2, 3-dihydronaphtho [1, 2-b] furans, Eur. J. Med. Chem., 2014, 86, 605-612.

20- L. Xia and Y. R. Lee, Regioselective synthesis of novel and diverse naphtho [1, 2-b] furan-3carboxamides and benzofuran-3-carboxamides by cascade formal [3+2] cycloaddition, RSC Adv., 2014, 4, 36905-36916.

21- C.-Y. Hsieh, P.-C. Tsai, C.-H. Tseng, Y. Chen, L.-S. Chang, and S.-R. Lin, Inhibition of EGF/EGFR activation with naphtho [1, 2-b] furan-4, 5-dione blocks migration and invasion of MDA-MB-231 cells, Toxicol. Vitr., 2013, 27, $1-10$.

22- Y. Chen et al., Discovery of N-(Naphtho [1, 2-b] Furan-5-Y1) Benzenesulfonamides as Novel Selective Inhibitors of Triple-Negative Breast Cancer (TNBC), Molecules, 2018, 23, 678.

23- S. A. Patil, R. Patil, L. M. Pfeffer, and D. D. Miller, Chromenes: potential new chemotherapeutic agents for cancer, Future Med. Chem., 2013, 5, 1647-1660.

24- Y. Dong, K. Nakagawa-Goto, C.-Y. Lai, S. L. Morris-Natschke, K. F. Bastow, and K.-H. Lee, Antitumor agents 278. 4-Amino-2H-benzo [h] chromen-2-one (ABO) analogs as potent in vitro anti-cancer agents, Bioorg. Med. Chem. Lett., 2010, 20, 4085-4087.

25- Y. Dong, K. Nakagawa-Goto, C.-Y. Lai, S. L. Morris-Natschke, K. F. Bastow, and K.-H. Lee, Antitumor agents 281. Design, synthesis, and biological activity of substituted 4-amino-7, 8, 9, 10-tetrahydro-2H-benzo [h] chromen-2-one analogs (ATBO) as potent in vitro anticancer agents, Bioorg. Med. Chem. Lett., 2011, 21, 546-549.

26- Y. Dong et al., Antitumor agents. 272. StructureActivity relationships and in vivo selective antibreast cancer activity of novel neo-tanshinlactone analogues, J. Med. Chem., 2010, 53, 2299-2308.

27- C. T. Supuran and A. Scozzafava, Carbonic anhydrase inhibitors, Curr. Med. Chem. Endocr. Metab. Agents, 2001, 1, 61-97.

characterization and antimicrobial activity of novel xanthene sulfonamide and carboxamide derivatives, J. Enzyme Inhib. Med. Chem., 2013, 28, 885-893.

30- A. Scozzafava, C. T. Supuran, and F. Carta, 
Antiobesity carbonic anhydrase inhibitors: literature and patent review, Expert Opin. Ther. Pat., 2013, 23, 725-735.

31- B. Żołnowska, J. Sławiński, A. Pogorzelska, J. Chojnacki, D. Vullo, and C. T. Supuran, Carbonic anhydrase inhibitors. Synthesis, and molecular structure of novel series $\mathrm{N}$-substituted N'-(2-arylmethylthio-4-chloro-5methylbenzenesulfonyl) guanidines and their inhibition of human cytosolic isozymes I and II and the transmembrane tumor-associa, Eur. J. Med. Chem., 2014, 71, 135-147.

32- S. Bag et al., Sulfonamides as multifunctional agents for Alzheimer's disease, Bioorg. Med. Chem. Lett., 2015, 25, 626-630.

33- M. S. A. El-Gaby, M. M. Ghorab, Z. H. Ismail, S. M. Abdel-Gawad, and H. M. Aly, Synthesis, structural characterization and anticancer evaluation of pyrazole derivatives, Med. Chem. Res., 2018, 27, 72-79.

34- M. M. Ghorab, M. S. Alsaid, M. S. A. El-Gaby, N. A. Safwat, M. M. Elaasser, and A. M. Soliman, Biological evaluation of some new $\mathrm{N}$-(2, 6-dimethoxypyrimidinyl) thioureido benzenesulfonamide derivatives as potential antimicrobial and anticancer agents, Eur. J. Med. Chem., 2016, 124, 299-310.

35- M. M Ghorab et al., Novel thiourea derivatives bearing sulfonamide moiety as anticancer agents through COX-2 inhibition, Anti-Cancer Agents Med. Chem. (Formerly Curr. Med. Chem. Agents), 2017, 17, 1411-1425.

36- M. M. Ghorab, M. S. A. El-Gaby, A. M. Soliman, M. S. Alsaid, M. M. Abdel-Aziz, and M. M. Elaasser, Synthesis, docking study and biological evaluation of some new thiourea derivatives bearing benzenesulfonamide moiety, Chem. Cent. J., 2017, 11, 42.

37- M. M. Ghorab, M. S. Alsaid, M. S. A. El-Gaby, M. M. Elaasser, and Y. M. Nissan, Antimicrobial and anticancer activity of some novel fluorinated thiourea derivatives carrying sulfonamide moieties: synthesis, biological evaluation and molecular docking, Chem. Cent. J., 2017, 11, 32.

38- A. M. El-Agrody, A.-A. M. Al-Dies, and A. M. Fouda, Microwave-assisted synthesis of 2-amino6-methoxy-4H-benzo [h] chromene derivatives, Eur. J. Chem., 2014, 5, 133-137.

39- R.-A. Tucaliuc, V. V Cotea, M. Niculaua, C. Tuchilus, D. Mantu, and I. I. Mangalagiu, New pyridazine-fluorine derivatives: Synthesis, chemistry and biological activity. Part II, Eur. J. Med. Chem., 2013, 67, 367-372.

40- H.-A. S. Abbas, S. S. A. El-Karim, E. M. Ahmed, A. F. Eweas, and S. A. El-Awdan, Synthesis, biological evaluation and molecular docking studies of aromatic sulfonamide derivatives as anti-inflammatory and analgesic agents, Acta Pol. Pharm., 2016, 73, 1163-1180.

41- M. Gupta, R. Gupta, and M. Anand, Hydroxyapatite supported caesium carbonate as a new recyclable solid base catalyst for the Knoevenagel condensation in water, Beilstein J. Org. Chem., 2009, 5, 1-7.

42- M. Mittelbach, An improved and facile synthesis of 2-amino-1, 1, 3-tricyanopropene, Monatshefte für Chemie/Chemical Mon., 1985, 116, 689-691.

43- E. Committee, S. Testing, C. Microbiology, and I. D. Escmid, Determination of minimum inhibitory concentrations (MICs) of antibacterial agents by agar dilution, Clin. Microbiol. Infect., 2000, 6, 509-515.

44- NCCLS, Methods for Dilution Antimicrobial Susceptibility Tests for Bacteria That Grow Aerobically M7-A5. 5th ed. National Committee for Clinical Laboratory Standards; Wayne, PA, USA: 2000. Wayne (PA): The Committee, 2000.

45- M. A. Abdelrahman, I. Salama, M. S. Gomaa, M. M. Elaasser, M. M. Abdel-Aziz, and D. H. Soliman, Design, synthesis and 2D QSAR study of novel pyridine and quinolone hydrazone derivatives as potential antimicrobial and antitubercular agents, Eur. J. Med. Chem., 2017, $138,698-714$. 